\title{
A Hybrid Pulse Transformer With Permanent Magnets
}

\author{
Tsutomu Mizuno, Senior Member, IEEE, Yukinobu Takata, and Masashi Matsumoto \\ Faculty of Engineering, Shinshu University, Nagano 380-8553, Japan
}

\begin{abstract}
This paper compares the operating range of a conventional pulse transformer (PT) and a hybrid pulse transformer (HPT) which is designed for miniaturization and increased output power of flyback converters. The HPT expands the operating range of the primary magnetomotive force by impressing the dc bias in cores with permanent magnets. The operating ranges of the conventional PT and the HPT are, respectively, 32 and $54 \mathrm{~A}$, the operating range of the HPT is 1.7 times that of the conventional PT. The maximum output powers $P_{2}{ }_{m}$ of the conventional PT and the HPT are proportional to the square of their respective operating ranges. The $P_{2}{ }_{m}$ values of the conventional PT and the HPT are, respectively, 8.6 and $23.2 \mathrm{~W}$, the $P_{2} m$ of the HPT is 2.7 times that of the conventional PT.
\end{abstract}

Index Terms-Dc bias, flyback converter, hybrid pulse transformer (HPT), magnetomotive force, output power, permanent magnet (PM).

\section{INTRODUCTION}

$\mathbf{P}$ ULSE TRANSFORMERs (PTs) used in switching power supplies are desired for miniaturization and high output power. The PT for a switching power supply has realized miniaturization and high output power by high-frequency switching [1]. However, the core loss in conventional PTs increases with high-frequency switching, and there are many problems regarding miniaturization and high output power.

A current-limiting device [2], a transformer for high-voltage generator [3], and an ignition coil [4] were studied to improve the output characteristics of the transformer by impressing the $\mathrm{dc}$ bias in the cores. There is no research on the improvement of the output characteristics by impressing the dc bias in the cores of a transformer for a flyback converter, to our knowledge.

\section{StRUCtURE AND OPERATION PRINCIPLE OF HPT}

\section{A. Structure of HPT}

Fig. 1 shows the basic structure of the HPT. The HPT consists of two E-type ferrite cores (TDK, PC40) [6], four ferrite permanent magnets (PMs) (TDK, FB1A) [7] and windings. The flux $\Phi_{i}$ generated by the primary current $i_{1}$ and the flux $\Phi_{m}$ generated by PMs are shown in Fig. 1. The operating range of the primary magnetomotive force $N_{1} i_{1}$ is expanded by the dc bias due to $\Phi_{m}$. The width $w_{m}$ of PMs are changed to clarify the magnetomotive force and the output power. The structure of due to $\Phi_{m}$. The width $w_{m}$ of PMs are changed to clarify the HPT without PMs is defined as that of a conventional PT.

\section{B. Operating Range and Output Power}

Fig. 2 shows the operating range of the conventional PT and the HPT. The conventional PT shown in Fig. 2(a) uses only the primary quadrant of the $B-H$ curve. The HPT in Fig. 2(b) expands the operating range $N_{1} I_{1 p}$ of the primary magnetomotive force by impressing $\Phi_{m}$ for $\Phi_{i}$ due to $i_{1}$. As a result, the HPT

Digital Object Identifier 10.1109/TMAG.2005.855166

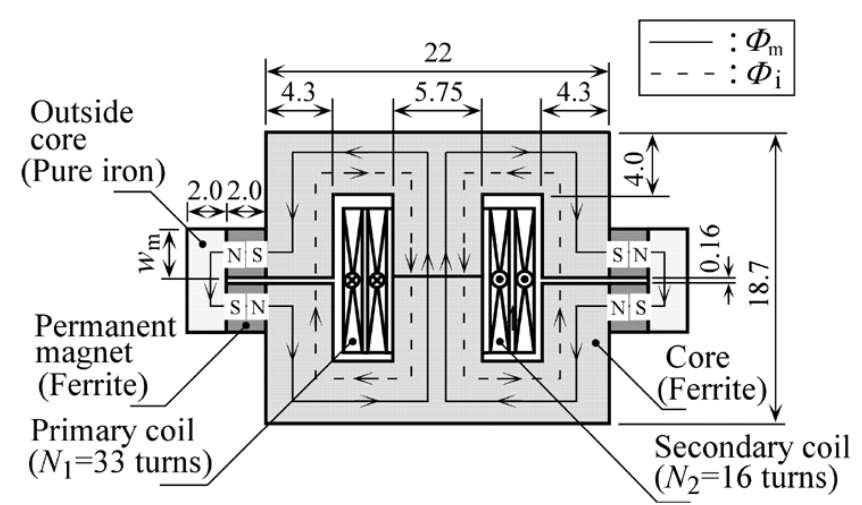

Fig. 1. Basic structure of HPT (unit: millimeters).

can have a primary magnetomotive force larger than that of the conventional PT.

The relationship between the operating range and the output power of the conventional PT and the HPT is described for a flyback converter operating in continuous mode. The maximum input power $P_{1 m}$ of the conventional PT and the HPT is given by [8]

$$
P_{1 m}=\frac{1}{2} f v_{1} i_{1 p} t_{\mathrm{on}} \quad(W)
$$

where $f$ is the frequency $(\mathrm{Hz}), v_{1}$ is the primary voltage $(V), i_{1 p}$ is the maximum primary current (A), and $t_{\text {on }}$ is the on time (s).

The maximum output power $P_{2 m}$ can be written as

$$
P_{2 m}=\eta P_{1 m} \quad(W)
$$

where $\eta$ is the efficiency.

The maximum output power $P_{2 m}$ is derived using (1) and (2) as

$$
P_{2 m}=\frac{1}{2} \eta \frac{f L_{1}}{N_{1}^{2}}\left(N_{1} i_{p}\right)^{2} \quad(W) .
$$

Therefore, the maximum output powers $P_{2 m}$ of the conventional PT and the HPT are proportional to the square of the operating range $N_{1} i_{1 p}$ of magnetomotive force if $L_{1}$ and $\eta$ are constant. 


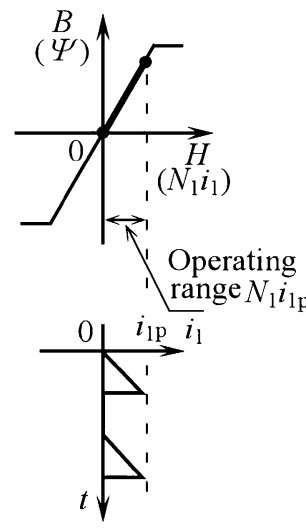

(a)

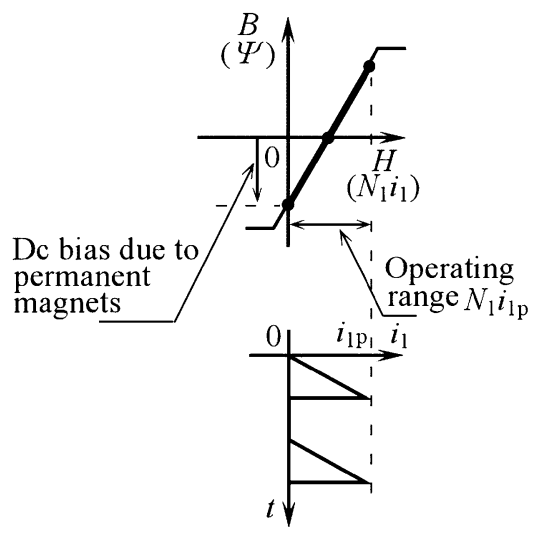

(b)
Fig. 2. Operating range of conventional PT and HPT. (a) Conventional PT. (b) HPT.

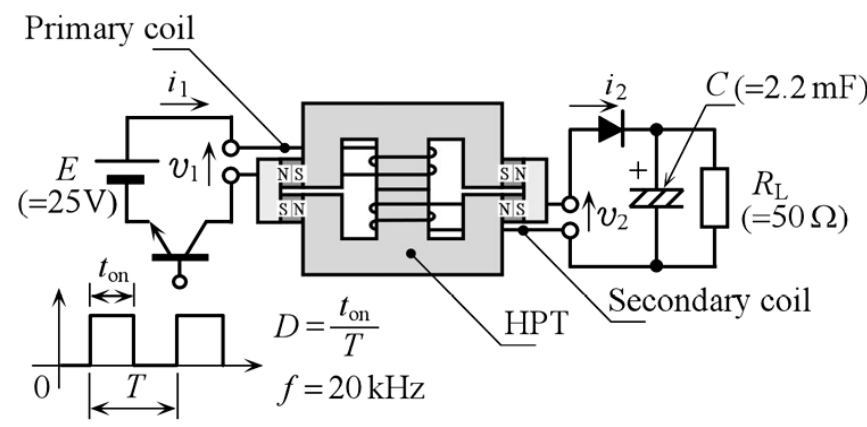

Fig. 3. Basic construction of flyback converter using HPT.

\section{EXPERIMENTAL CONDITIONS}

Fig. 3 shows the basic construction of a flyback converter using the HPT. Because the flyback converter is composed of a simple circuit and can be fabricated cheaply, the flyback converter is used as a small switching regulator. The flyback converter is composed of a switching transistor in the primary side, a diode and a condenser in the secondary side. $f$ is $20 \mathrm{kHz}$, the load resistance $R_{L}$ is $50 \mathrm{~W}$, the applied voltage $E$ is $25 \mathrm{~V}$, and the capacitance $C$ is $2.2 \mathrm{mF}$. The output characteristics were measured by changing the duty factor $D$.

\section{Measurement Results}

Fig. 4 shows the measured flux density characteristics of the conventional PT and the HPT measured with direct current magnetic characteristic measure apparatus. The operating ranges $N_{1} i_{1 p}$ of the conventional PT and the HPT with PMs of $w_{m}=5$ $\mathrm{mm}$ are, respectively, 32 and $54 \mathrm{~A}$, the operating range of the HPT with PMs of $w_{m}=5 \mathrm{~mm}$ is 1.7 times that of the conventional PT.

Fig. 5 shows the measured waveforms of the conventional PT and the HPT. Under the linear region of the magnetic circuit, primary current increases linearly. When magnetic saturation occurs in the core, $i_{1}$ rapidly increases. The maximum primary current $i_{1 p}$ was measured under the linear region of the magnetic circuit. The maximum primary currents $i_{1 p}$ of the conventional PT and the HPT with PMs of $w_{m}=5 \mathrm{~mm}$ were, respectively, 1.1 and $1.7 \mathrm{~A}$. The operating ranges $N_{1} i_{1 p}$ of the magnetomotive forces of the conventional PT and the HPT with PMs

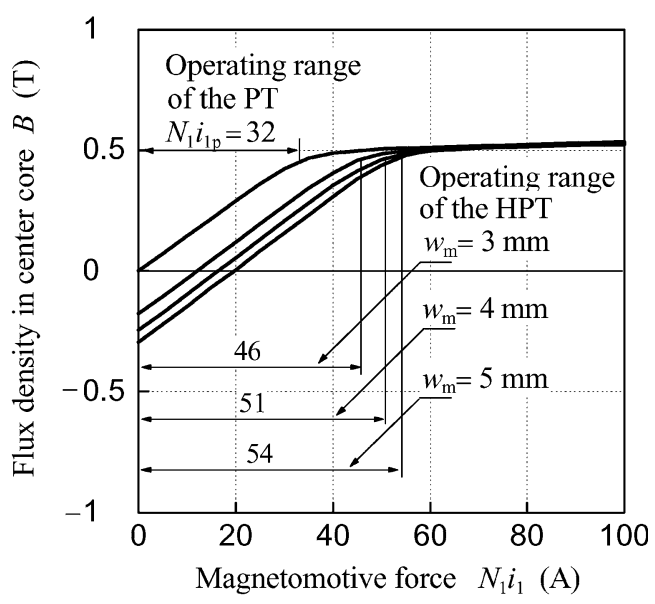

Fig. 4. Flux density characteristics of conventional PT and HPT.

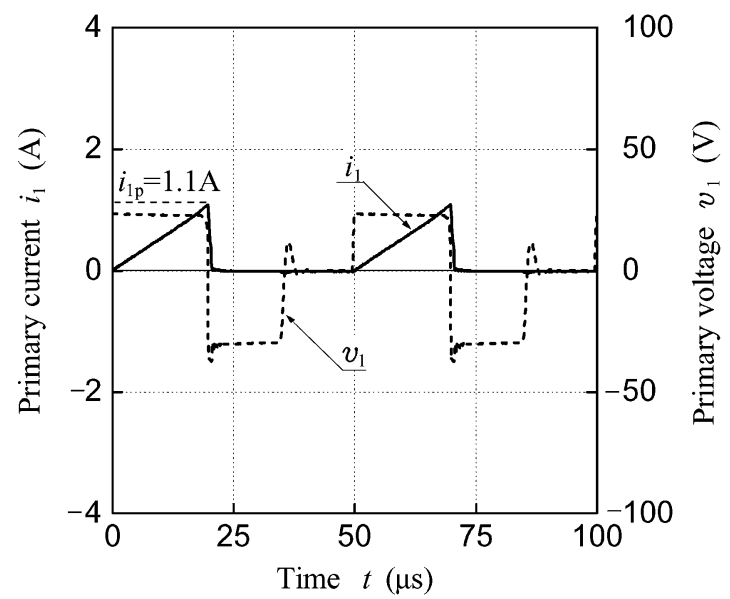

(a)

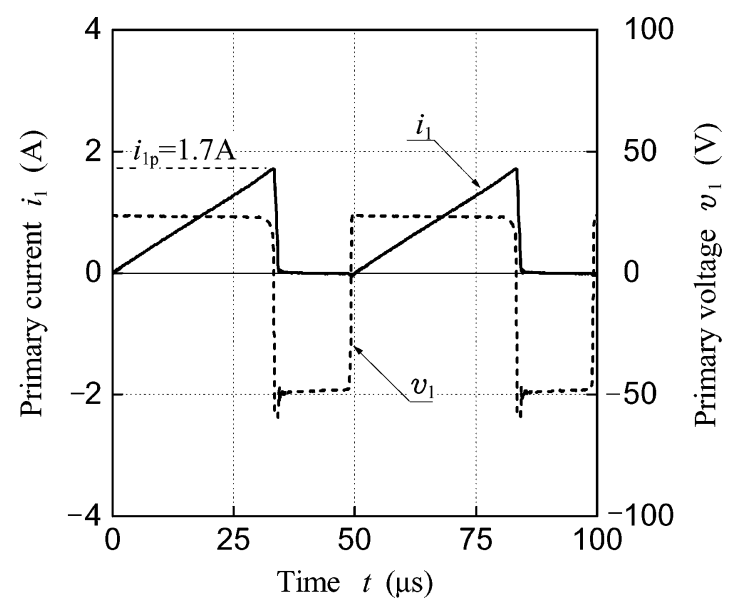

(b)

Fig. 5. Primary current and voltage waveforms of conventional PT and HPT. (a) Conventional PT ( $f=20 \mathrm{kHz}, E=25 \mathrm{~V}, R_{L}=50 \mathrm{~W}, D=0.4$ ). (b) HPT $\left(f=20 \mathrm{kHz}, E=25 \mathrm{~V}, R_{L}=50 \mathrm{~W}, D=0.65, w_{m}=5 \mathrm{~mm}\right)$.

of $w_{m}=5 \mathrm{~mm}$ were, respectively, 35 and 57 A because the number of turns of the primary coil $N_{1}$ was 33 . The operating range $N_{1} i_{1 p}$ of the HPT with PMs of $w_{m}=5 \mathrm{~mm}$ was 1.6 times that of the conventional PT. The operating range $N_{1} i_{1 p}$ of the conventional PT and the HPT obtained using Fig. 5 are almost the same as those obtained using Fig. 4. 


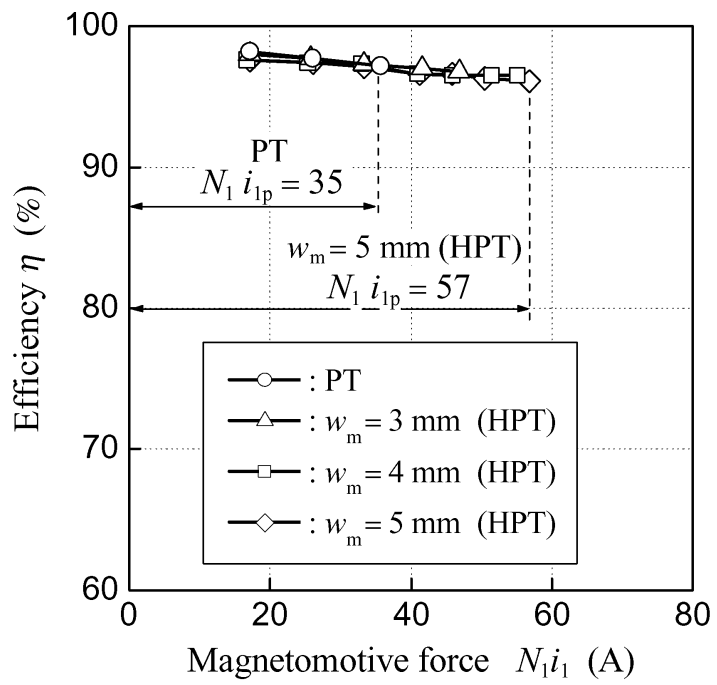

Fig. 6. Efficiency characteristics of conventional PT and HPT ( $f=20 \mathrm{kHz}$, $\left.E=25 \mathrm{~V}, R_{L}=50 \mathrm{~W}\right)$. The operating range $N_{1} i_{1 p}$ of the magnetomotive force is obtained using the primary current waveforms shown in Fig. 5.

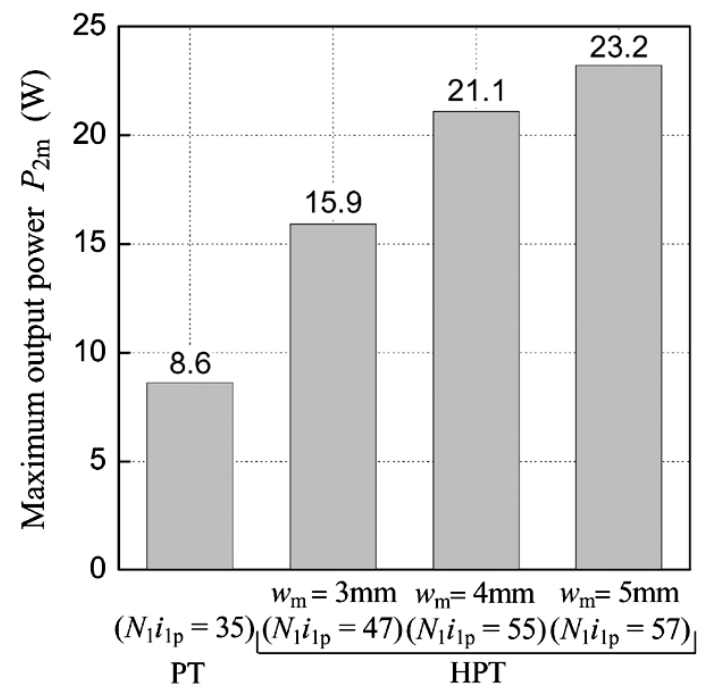

Fig. 7. Maximum output power characteristics of conventional PT and HPT ( $f=20 \mathrm{kHz}, E=25 \mathrm{~V}, R_{L}=50 \mathrm{~W}$ ). The operating range $N_{1} i_{1 p}$ of the magnetomotive force is obtained using the primary current waveforms shown in Fig. 5.

Fig. 6 shows the efficiency characteristics of the conventional PT and the HPT. The efficiency decreased with increasing primary magnetomotive force $N_{1} i_{1}$.
Fig. 7 shows the maximum output power characteristics of the conventional PT and the HPT. The maximum output powers $P_{2 m}$ of the conventional PT and the HPT with PMs of $w_{m}=5$ $\mathrm{mm}$ were, respectively, 8.6 and $23.2 \mathrm{~W}$, the $P_{2 m}$ of the HPT was 2.7 times that of the conventional PT.

The relationship between the operating range and the maximum output power is considered. The ratio $K$ of the $P_{2 m}$ of the HPT to that of the conventional PT was 2.7 as above. The operating range $N_{1} i_{1 p}$ of the conventional PT and the HPT with PMs of $w_{m}=5 \mathrm{~mm}$ were, respectively, 32 and $54 \mathrm{~A}$ as shown in Fig. 4. The ratio $K$ can be calculated as 2.8 using (3). The calculated $K$ is almost equivalent to the measured $K$.

\section{CONCLUSION}

This paper described the operating range of the primary magnetomotive force and the maximum output characteristics of the conventional PT and the HPT. The following results were obtained.

1) The operating range of the primary magnetomotive force was expanded by impressing the dc bias in the cores with PMs. The operating ranges of the conventional PT and the HPT with PMs of $w_{m}=5 \mathrm{~mm}$ were, respectively, 35 and 57 A as shown in Fig. 6, the operating range of the HPT was 1.6 times that of the conventional PT.

2) The $P_{2 m}$ values of the conventional PT and the HPT with PMs of $w_{m}=5 \mathrm{~mm}$ were, respectively, 8.6 and $23.2 \mathrm{~W}$, the $P_{2 m}$ of the HPT was 2.7 times that of the conventional PT.

\section{REFERENCES}

[1] Handbook of Switching Power Supply, Daily Industries Newspaper Publishing, Tokyo, Japan, 1993.

[2] B. P. Raju, K. C. Parton, and T. C. Bartram, "A current limiting device using dc bias applications and prospects," IEEE Trans. Power App. Syst., vol. 9, pp. 3173-3177, Sep. 1982.

[3] Matsushita Electric Industrial Co., Ltd., Osaka, Japan, "Transformer for High Voltage Generating," Japanese Patent 196243A, Jun. 19, 2001.

[4] R. Bosch, "The Ignition Coil for Ignition Equipment of Internal Combustion," Japanese Patent S59-167006, Sep. 20, 1984.

[5] T. Mizuno, T. Ikeda, Y. Takata, M. Tamura, T. Ishikawa, and H. Yamada, "Consideration on the operation principle of a hybrid pulse transformer," in Proc. Technical Meet. Static Apparatus, 2003, IEE Jpn, SA-03-86, pp. $7-13$.

[6] TDK Corporation, Tokyo, Japan, Core Catalog, pp. 20-25, 2003.

[7] TDK Corporation, Tokyo, Japan, Magnet Catalog, TDK, pp. 10-13, 2003.

[8] J. Togawa, Design of Practical Power Supply Circuit. Tokyo, Japan: CQ Publishing, 1988.

Manuscript received February 3, 2005 